\title{
Objective Assessment of Cervical Stiffness after Administration of Misoprostol for Intrauterine Contraceptive Insertion
}

Authors

Affiliations

\section{S. Badir ${ }^{1}$ E. Mazza ${ }^{1,2}$, M. Bajka}

${ }^{1}$ Swiss Federal Institute of Technology Zurich, Institute of Mechanical Systems, Zurich, Switzerland ${ }^{2}$ EMPA, Swiss Federal Laboratories for Materials Testing and Research, Dübendorf, Switzerland ${ }^{3}$ University Hospital Zurich, OB/GYN, Zurich, Switzerland

\section{Key words}

cervical stiffness

- cervical softening

- cervical priming

- misoprostol

- aspiration technique received 27.10.2015

accepted 22.03.2016

Bibliography

DOI http://dx.doi.org/

10.1055/s-0042-106393

Published online: 2016

Ultrasound International Open

2016; 2: E63-E67

(c) Georg Thieme Verlag KG

Stuttgart · New York

ISSN 2199-7152

\section{Correspondence}

\section{Prof. M. Bajka}

University Hospital Zurich

OB/GYN

Frauenklinikstrasse 10

8091 Zurich

Switzerland

Tel.: +41/79/6295181

michael.bajka@hin.ch

\section{Abstract}

$\nabla$

Purpose: The goal of this study was to objectively quantify cervical stiffness in misoprostol users prior to IUC insertion and at follow-up consultation to evaluate the feasibility of assessing cervical stiffness and to study the influence of misoprostol on cervical softening.

Materials and Methods: This was a crosssectional study that evaluated 40 women who wished to use the LNG IUS. These women were evaluated immediately before LNG IUS insertion and 6 weeks later at follow-up consultation. Participants received $200 \mu \mathrm{g}$ of misoprostol combined with $75 \mathrm{mg}$ of diclofenac in a single tablet orally (Arthrotec forte $75 / 200^{\circledR}$, Pfizer, USA) $6-12 \mathrm{~h}$ prior to insertion in "off label" use. On both occasions, cervical stiffness was determined

\section{Introduction}

Intrauterine contraceptives (IUC) are among the most widely used family planning methods worldwide. Modern IUCs are reversible, longacting, safe and cost-effective [1]. However, IUC uptake varies significantly across the world. Fear of pain during insertion is one of the well-known reasons that may prevent women from choosing an IUC as their method of choice $[2,3]$. For the vast majority, IUC insertion does not cause severe pain. However, around $10 \%$ of (multi-)para (P) [4] and $20 \%$ of nullipara (NP) [5] women report this procedure to be very painful. It may, therefore, seem surprising that the need for pain relief during IUC insertion is still under debate and there is no generally accepted concept.

Application of misoprostol, a prostaglandin analog, activates inflammatory reactions [6,7] and induces microstructural changes in the cervical tissue leading to a disorganization of the collagenous network and thus cervical tissue softening (sometimes also called cervical ripening) $[8,9]$. using a novel medical device based on the aspiration technique. The Wilcoxon rank-sum and the Wilcoxon signed-rank test were applied to compare cervical stiffness assessments at insertion of the IUD and at follow-up.

Results: For the first time, cervical stiffness was quantitatively assessed in misoprostol users prior to IUD insertion, proving that the aspiration technique enables detection of pharmacologically induced cervical changes, and also that misoprostol has a detectable softening effect on cervical tissue.

Conclusion: The clinical value of the detected cervical softening after misoprostol administration remains unclear. Aspiration measurements could be helpful in searching for the ideal candidate, the appropriate route, dosage and interval of misoprostol intake prior to IUC insertion.
In $\mathrm{OB} / \mathrm{GYN}$, misoprostol has been widely administered in "off-label" usage to initiate the expected cervical softening as part of easing cervical dilation $[4,10,11]$. However, the cervical softening which is an indirect clinical feature and a requisite to facilitate cervical manipulations has not yet been quantified in-vivo.

The never proven softening effect of misoprostol was also evaluated for pain reduction in IUC insertion [3], especially with respect the possible advantage for NP, who generally present a narrow, stiff and un-stretched cervix $[10,12,13]$. Indeed, some studies found the use of misoprostol leads to an easier IUC passage through the cervical canal [14-16] and greater cervical dilation [15], but several other studies could not support the superiority of misoprostol administration, neither from the doctor's judgment regarding ease of insertion $[4,17,18]$ nor from the patient's judgment regarding lower pain perception $[4,17]$. In a large review, Gemzell-Danielsson et al. [19] found no conclusive evidence that prophylactic pharmacological interventions 
reduce pain on IUC insertion. This conflicting data, doubting the effectiveness of misoprostol, might arise from a lack of a standardized protocol for IUC insertion and drug application (route, time dosage) [19-22]. However, despite the lack of clear evidence of a beneficial effect of misoprostol, health care providers frequently recommend misoprostol administration in the belief that pain during IUC insertion is reduced [23].

The state of scientific knowledge regarding the efficacy of different misoprostol regimens on cervical softening and on IUC insertion in general needs further research. The quantitative and objective assessment of cervical softening using a dedicated instrument is the first step towards an improved understanding. Such an instrument makes it possible to find the optimal misoprostol treatment in terms of cervical softening which then can be further evaluated in terms of ease of insertion and impact on pain management during IUC insertion.

In the present study, we focused on acquiring objective data to measure cervical stiffness using the aspiration method (ASP) [24]. Recently, the aspiration method has been tested successfully in a clinical study to describe cervical stiffness in 50 nonpregnant and 50 pregnant subjects. For the first time the in-vivo measurements allowed us to quantify the continuous cervical softening during pregnancy $[24,25]$. To the best of our knowledge, aspiration is the only available and reliable method that allows us to objectively quantify the stiffness of cervical tissue in humans in-vivo.

The goal of this study is to objectively quantify cervical stiffness in misoprostol users prior to IUC insertion and at follow-up consultation to evaluate the feasibility of assessing cervical stiffness and to study the influence of misoprostol on cervical softening.

\section{Materials and Methods}

$\nabla$

\section{Ethical approval}

This cross-sectional study was approved by the IRB (KEK Zurich StV02/2007 and later amendments) and was conducted according to the Declaration of Helsinki. Subject informed consent was a prerequisite for study involvement.

\section{Participant selection}

From July 2013 all women presenting for IUC placement at our private office were invited to take part in the study. We focused on the $52 \mathrm{mg}$ LNG IUS (LNG IUS, Mirena ${ }^{\circledR}$, Bayer Healthcare, Germany) since more than $90 \%$ of the women chose an LNG IUS as their IUC in this unit. Exclusion criteria were communication problems, prior surgery on the cervix, untreated premalignant or malignant changes on the cervix, contraindications to using an LNG IUS, and the use of misoprostol or non-steroidal antiinflammatory drugs. By October 2014, 40 women had been included in the study.

\section{Misoprostol and Diclofenac administration}

Participants received $200 \mu \mathrm{g}$ of misoprostol combined with $75 \mathrm{mg}$ of diclofenac in a single tablet orally (Arthrotec forte $75 / 200^{\circledR}$, Pfizer, USA) 6-12 h before insertion in "off label” use.

\section{Aspiration measurement}

Directly before LNG IUS insertion, aspiration measurements ( $\bullet$ Fig. 1) were performed, as previously described in detail $[24,25]$ to measure cervical stiffness. To this end, the aspiration tube is placed orthogonally on the ecto-cervix at the 12 o'clock position. Negative pressure (limited at maximum 500 mbar) on the cervical epithelium is applied to elevate the underlying tissue to a predefined level of deformation $(4 \mathrm{~mm})$. The required pressure to deform the tissue (closure pressure: $\mathrm{p}_{\mathrm{cl}}$ ) is registered. It is proportional to the stiffness of the cervical stroma $[24,25]$.

\section{Follow-up}

Aspiration measurements were repeated at regular follow-up consultations including a sonographic IUC position check around 6 weeks after insertion.

\section{Study population}

The study included 40 Caucasians aged from 22 to 49 years of age (mean: 35 years), mostly parous subjects. 25 subjects underwent a first LNG IUS insertion, and 15 subjects had an LNG IUS replacement.

\section{Statistics}

Statistical analysis was performed with the statistical computing environment R: A Language and Environment for Statistical Computing, Open Source Software, 2012. The Wilcoxon ranksum test was used to compare differences between the previously investigated control group [24] and the 2 groups in this study, both prior to IUC placement (first insertion and replacement) and at follow-up. For comparison of values of the same subjects at insertion and at follow-up, the Wilcoxon signed-rank test was applied.

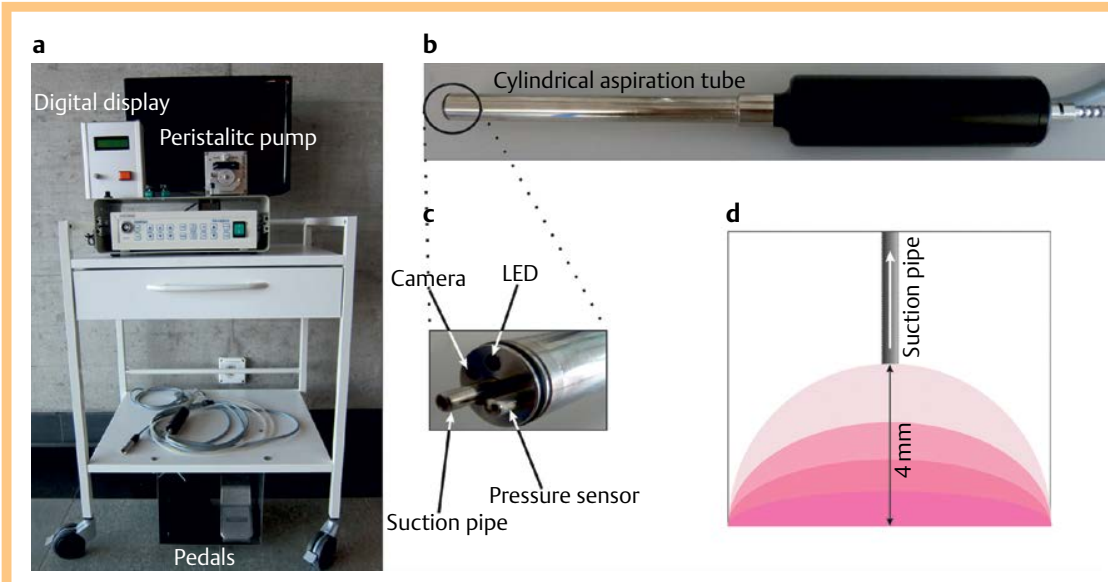

Fig. 1 Aspiration device and measurement principle. a Trolley with peristaltic pump, aspiration tube, pedals, and other technical equipment. b View of the cylindrical aspiration tube with the round contact opening (diameter of $8 \mathrm{~mm}$ ) at the tip is placed at 12 o'clock position on the cervix. c Inside of the aspirator head. $\mathbf{d}$ The pressure in the tube is reduced by extraction of air through the suction pipe. Cervical tissue is deformed into the aspiration tube until the tissue vault reaches and closes the suction pipe. The corresponding value of negative pressure is called the closing pressure $\left(p_{c}\right)$ and is the output of the measurement 


\section{Results}

All new LNG IUS insertions as well as all replacements could be executed successfully. Immediate transvaginal ultrasound position check confirmed correct positioning in all cases, and no perforation occurred.

Cervical stiffness $\left(\mathrm{p}_{\mathrm{cl}}\right)$ was successfully assessed in all subjects. The primary outcome was the $\mathrm{p}_{\mathrm{cl}}$ value (i.e., cervical stiffness) at insertion after misoprostol intake and at follow-up without misoprostol. We found average values for $\mathrm{p}_{\mathrm{cl}}$ of $290 \mathrm{mbar} \pm 138 \mathrm{mbar}$ (mean \pm SD) at insertion and $324 \pm 138$ mbar at follow-up showing no significant difference when assessing the group as a whole. However, when we divided the study population into first LNG IUS insertion and LNG IUS replacement, we found 230 mbar \pm 93 mbar (mean $\pm S D$ ) in the first LNG IUS insertion group $(\mathrm{n}=25)$, and $\mathrm{p}_{\mathrm{cl}}$ of $396 \mathrm{mbar} \pm 90$ mbar in the LNG IUS replacement group $(n=15)$. The comparison with historical control subjects $(n=50)$ published in Badir et al. [25] (320 mbar \pm 120 mbar) revealed that $\mathrm{p}_{\mathrm{cl}}$ at insertion was significantly lower $(\mathrm{p}=0.005)$ in the first LNG IUS insertions, indicating a reduction in cervical stiffness. In contrast, the comparison between control subjects and LNG IUS replacement demonstrated a higher $\mathrm{p}_{\mathrm{cl}}$ in this group, but did not reach statistical significance $(p=0.08)$. Additionally, comparison of $\mathrm{p}_{\mathrm{cl}}$ values at insertion between first insertions and replacements showed a significant difference $(\mathrm{p} \leq 0.001)$. Pre- and post-comparison of $\mathrm{p}_{\mathrm{cl}}$ at insertion and at follow-up demonstrated a differentiated behavior in cervical stiffness change in the 2 groups. In the first LNG IUS insertion group, cervical stiffness increased significantly $(\mathrm{p} \leq 0.001)$ to a similar level ( 308 mbar \pm 105 ) at follow-up as that expected from the control group ( 320 mbar \pm 120 mbar) while in the LNG IUS replacement group cervical stiffness decreased significantly to the level of the control group ( $p=0.03$ ). See $\bullet$ Fig. 2 for mean and standard deviation values and $\bullet$ Fig. $\mathbf{3}$ for subject-specific data. Closure pressure values obtained at follow-up are not statistically different from the control group (first insertion, $\mathrm{p}=0.6$ and consecutive insertion, $\mathrm{p}=0.7$ ). The same non-significant finding is revealed in the comparison between $\mathrm{p}_{\mathrm{cl}}$ values between follow-up after first LNG IUS placement and LNG IUS replacement $(\mathrm{p}=0.5)$.

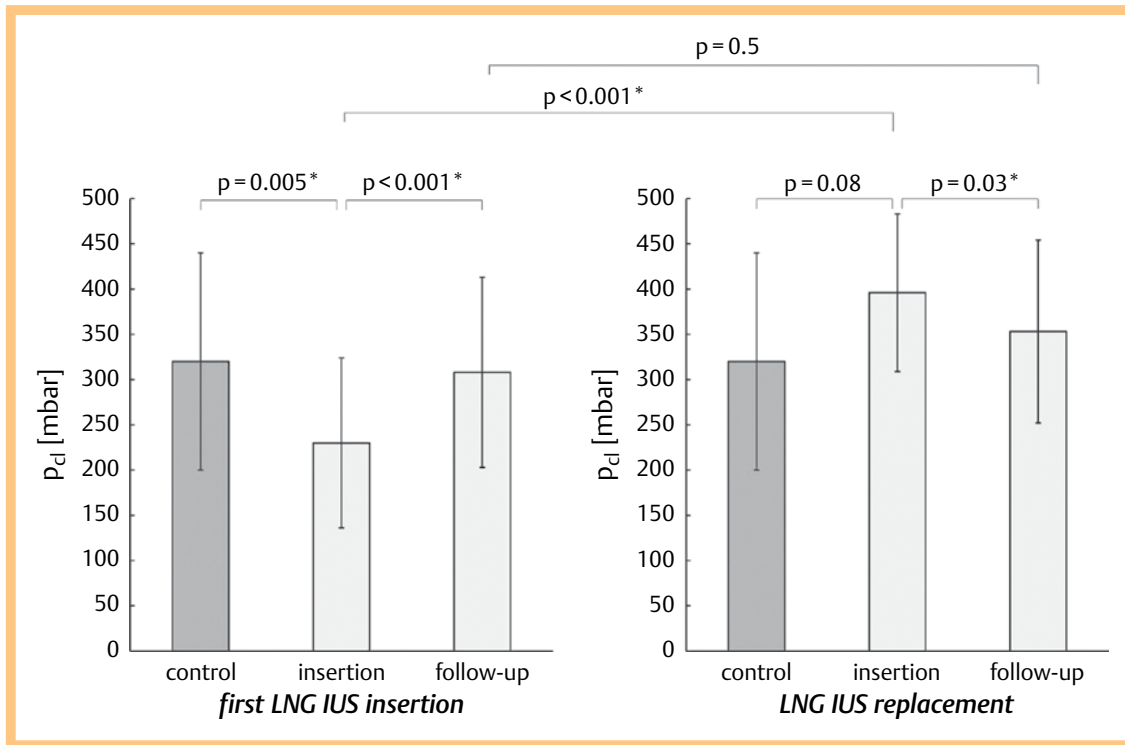

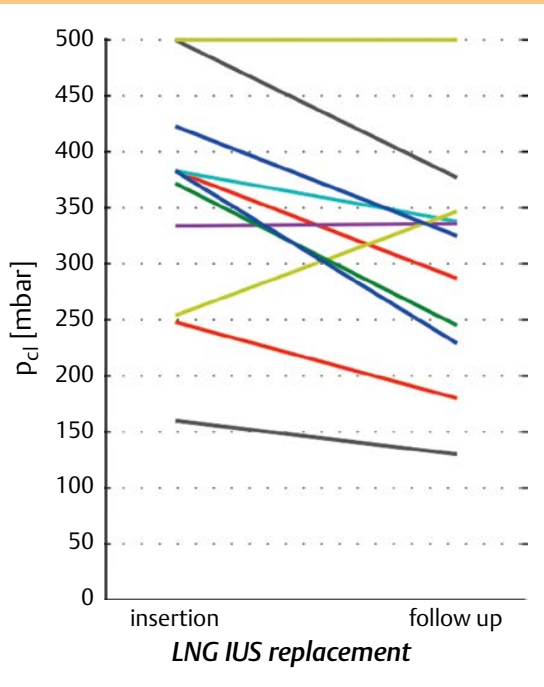

Fig. 2 Results of closure pressure $\mathrm{P}_{\mathrm{cl}}$ (cervical stiffness) of the control group [25] and subjects at insertion and at follow-up. a First LNG IUS insertions. $\mathbf{b}$ LNG IUS replacement. For all values, means and standard deviations and $p$-values are reported. Significant results are indicated by ${ }^{*}(p<0.05)$.

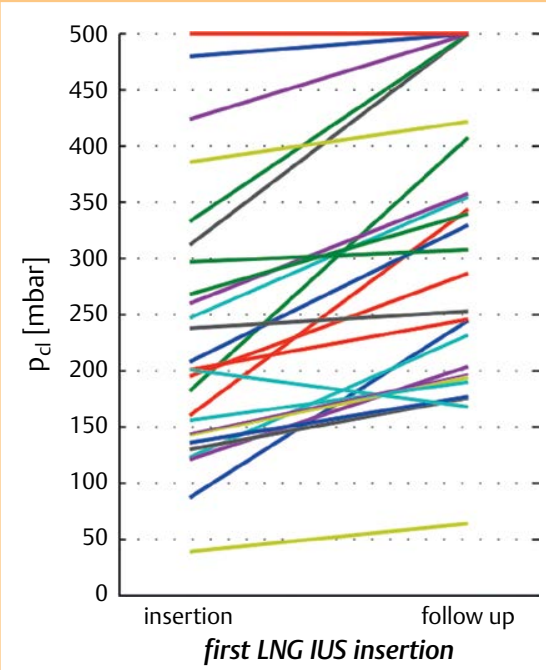

first LNG IUS insertion
Fig. 3 Pre- and post-comparison of closure pressure $\mathrm{P}_{\mathrm{cl}}$ (cervical stiffness) of each subject individually at insertion and at follow-up. a First LNG IUS insertion. $\mathbf{b}$ LNG IUS replacement. 


\section{Discussion}

$\nabla$

For the first time, cervical stiffness was quantitatively assessed in misoprostol users prior to LNG IUS insertion, showing that i) ASP is able to detect pharmacologically induced cervical changes, and ii) misoprostol has a detectable softening effect on cervical tissue at first insertion.

We compared measurements taken from women at their first LNG IUS insertion with their measurements taken around 6 weeks later (when there were no effects from the previous misoprostol administration). It was found that cervical stiffness was significantly lower initially with misoprostol but after 6 weeks cervical stiffness recovered to the reference stiffness values of non-pregnant women.

In contrast, the group with an LNG IUS replacement did not show a decrease of cervical softening caused by misoprostol. Cervical stiffness was not significantly different at insertion and at follow-up some weeks later, and was comparable to the reference cohort. These results are in line with Heikinheimo et al. [4]. In their study misoprostol did not have an effect on the ease of insertion in subjects having an LNG IUS replacement.

This surprise finding requires further explanation. We can only assume that prostaglandin (misoprostol)-induced cervical softening was blocked in the LNG IUS exchangers by the locally released LNG. This assumption is supported by the recent literature. Murine infection-induced preterm birth models provided insight into the molecular and microstructural processes leading to cervical softening. Prostaglandins were found to be the promoter of these remodeling processes. Increased GAG synthesis induced by prostaglandins led to disintegration of the collagenous network, thus leading to detectable cervical softening. In these cases the softness of the cervical tissue was indistinguishable from that of cervices at term. Moreover, using the same murine model, it was demonstrated that administration of progesterone inhibited prostaglandin induced cervical softening [6].

In our study, $200 \mu \mathrm{g}$ misoprostol and $75 \mathrm{mg}$ diclofenac were given orally $6-12 \mathrm{~h}$ prior to insertion of the LNG IUS. The oral administration route was chosen for practical reasons combined with a smaller dose of misoprostol, as discussed by Sääv et al. [14], to lower the incidence of uterine cramps. Nonetheless, diclofenac was co-administered, as suggested by Gemzell-Danielsson et al. [19], to manage prostaglandin-induced side effects. In previous IUC insertion studies, misoprostol was administered bucally [10], sublingually [14] or vaginally [15]. In contrast to our protocol, the dosage of $400 \mu \mathrm{g}$ administered in the above mentioned studies was significantly higher and the time lag between misoprostol intake and insertion within 1-4h was significantly shorter $[4,10,17]$. We decided for a longer priming interval based on the recommendations of different authors $[11,14,21,22]$ who critically addressed the importance of the time interval to induce significant cervical softening and to obtain the benefit of misoprostol for insertion. Since we measured a significant softening after misoprostol intake in the first LNG IUS insertions, our observation supports the importance of a priming interval of $6-12 \mathrm{~h}$. However, this study was not designed to answer this question.

The exact role of diclofenac remains unclear, but we believe that the very low rate of misoprostol-induced uterine cramps (5\%) in this study is the result of the diclofenac effect, as assumed in Bahamondes et al. [22].
A limitation of this study is the lack of sub-analysis of the misoprostol effect on NP vs. P due to the small number of NP subjects, and the restriction to only one typically used IUC. Another possible limitation of this study is that it was not conducted as a blind randomized controlled trial and cervical softening is an indirect clinical feature that does not allow judgment e.g., about ease of insertion, extent of cervical dilation or pain during IUC insertion.

\section{Conclusion}

$\nabla$

The aspiration method allowed simple and quantitative cervical stiffness assessment to evaluate the softening effect of misoprostol on cervical tissue. Our results are indicative for misoprostol-induced softening in subjects for first LNG IUS insertion. As a preliminary clinical consequence, based on our results, we could suggest a differentiated misoprostol administration policy, i.e., women undergoing first LNG IUS insertion might benefit from $200 \mu \mathrm{g}$ oral misoprostol administration $6-12 \mathrm{~h}$ prior to insertion (evidence of cervical softening), while women undergoing LNG IUS replacement might not benefit from it (lack of cervical softening). However, cervical softening is an indirect clinical value and should be further studied by assessing clinical features such as ease of insertion or pain during insertion. Further aspiration measurements could be helpful in searching for the ideal candidate, the appropriate route, dosage and interval of misoprostol intake prior to IUC insertion.

\section{References}

1 Winner B, Peipert J, Qiuhong $Z$ et al. Effectiveness of Long-Acting Reversible Contraception. N Engl J Med 2012; 366: 1998-2007

2 Hubacher D, Reyes V, Lillo $S$ et al. Pain from copper intrauterine device insertion: randomized trial of prophylactic ibuprofen. Am J Obstet Gynecol 2006; 195: 1272-1277

3 Allen R, Bartz D, Da G et al. Interventions for pain with intrauterine device insertion. Cochrane Database Syst Rev 2009

4 Heikinheimo 0 , Inki P, Kunz $M$ et al. Double-blind, randomized, placebo-controlled study on the effect of misoprostol on ease of consecutive insertion of the levonorgestrel releasing intrauterine system. Contraception 2010; 81: 481-486

5 Marions L, Lövkvist L, Taube A et al. Use of the levonorgestrel releasingintrauterine system in nulliparous women - a noninterventional study in Sweden. Eur J Contracept Reprod Health Care 2011; 16: 126-134

6 Gonzalez J, Franzke C, Yang F et al. Complement activation triggers metalloproteinases release inducing cervical remodelling and preterm birth in mice. Am J Pathol 2011; 179: 838-848

7 Norman M, Ekman G, Malmström A. Prostaglandin E2-induced ripening of the human cervix involves changes in proteoglycan metabolism. Obstet Gynecol 1993; 82: 1013-1020

8 Akins M, Luby-Phelps $K$, Bank $R$ et al. Cervical softening during pregnancy: regulated changes in collagen crosslinking and composition of matricellular proteins in the mouse. Biol Reprod 2011; 84: 1053-1062

9 Myers KM, Feltovich H, Mazza E et al. The mechanical role of the cervix in pregnancy. J Biomech 2015; 48: $1511-1523$

10 Lathrop E, Haddad L, McWhorter CP et al. Self-administration of misoprostol prior to intrauterine device insertion among nulliparous women: a randomized controlled trial. Contraception 2013; 88: 725-729

11 Choksuchat C, Cheewadhanaraks S, Getpook C et al. Misoprostol for cervical ripening in non-pregnant women: a randomized double-blind controlled trial of oral versus vaginal regimens. Hum Reprod 2006; 21: $2167-2170$

12 Farmer $M$, Webb A. Intrauterine device insertion-related complications: can they be predicted? J Fam Plann Reprod Health Care 2003; 29: 227-231

13 Kaislasuo J, Suhonen S, Gissler $M$ et al. Uterine perforation caused by intrauterine devices: clinical course and treatment. Hum Reprod 2012; 27: 2658-2663 
14 Sääv I, Aronsson a, Marions L et al. Cervical priming with sublingual misoprostol prior to insertion of an intrauterine device in nulliparous women: a randomized controlled trial. Hum Reprod 2007; 22: 2647-2652

15 Scavuzzi A, Souza ASR, Costa R et al. Misoprostol prior to inserting an intrauterine device in nulligravidas: a randomized clinical trial. Hum Reprod 2013; 28: 2118-2125

16 Lotke PS, Tiwari A, Nuño VL. Inserting intrauterine devices in nulliparous women: is misoprostol beneficial? A registered clinical trial 2013; 1: 62-68

17 Espey E, Singh RH, Leeman L et al. Misoprostol for intrauterine device insertion in nulliparous women: a randomized controlled trial. Am J Obstet Gynecol 2014; 210: 1-5

18 Dijkhuizen K, Dekkers OM, Holleboom CG et al. Vaginal misoprostol prior to insertion of an intrauterine device: an RCT. Hum Reprod 2011; 26: 323-329

19 Gemzell-Danielsson K, Mansour D, Fiala C et al. Management of pain associated with the insertion of intrauterine contraceptives. Hum Reprod Update 2013; 19: 419-427
$20 \mathrm{Li} Y T$, Kuo TC, Kuan LC et al. Cervical softening with vaginal misoprostol before intrauterine device insertion. Int J Gynaecol Obstet 2005; 89: $67-68$

21 Li Y-T, Wen-Ling L, Peng-Hui W. Difficult intrauterine device insertion. Hum Reprod 2011; 26: 2192-2193

22 Bahamondes L, Mansour D, Fiala $C$ et al. Practical advice for avoidance of pain associated with insertion of intrauterine contraceptives. J Fam Plann Reprod Health Care 2014;40: 54-60. [182] Crane J. prime the cervix for hysteroscopy? Obstet Gynecol Manag 2008; 20: 27-29

23 Ward K, Jacobson JC, Turok DK et al. A survey of provider experience with misoprostol to facilitate intrauterine device insertion in nulliparous women. Contraception 2011; 84: 594-599

24 Badir S, Bajka M, Mazza E. A novel procedure for the mechanical characterization of the uterine cervix during pregnancy. J Mech Behav Biomed Mater 2013; 27: 143-153

25 Badir S, Mazza E, Zimmermann R et al. Cervical softening occurs early in pregnancy: characterization of cervical stiffness in 100 healthy women using the aspiration technique. Prenat Diagn 2013; 33: 737-741 